\title{
NUMERICAL SOLUTION OF THE LEADER PROGRESSION MODEL BY MEANS OF THE FINITE ELEMENT METHOD
}

\author{
A. Borghetti ${ }^{1}$, F. Napolitano ${ }^{1}$, C.A. Nucci ${ }^{1}$, M. Paolone ${ }^{1}$, M. Bernardi $^{2}$ \\ 1. University of Bologna \\ 2. CESI S.p.A. \\ alberto.borghetti@unibo.it, fabio.napolitano2@unibo.it, carloalberto.nucci@unibo.it,mario.paolone@unibo.it, \\ marina.bernardi@cesi.it
}

\begin{abstract}
As known the lightning incidence of a vertical structure is related to the physical processes involved in the final stage of the progression of the downward lightning leader to the structure and upward streamers, in case downward flashes are taken into account. Within this context, the paper presents and discusses a numerical implementation based on the Finite Element Method (FEM) of the Leader Progression Model (LPM): LPM-FEM. The obtained results are compared with those of the original implementation of the LPM based on the charge simulation method and the relevant differences analyzed and discussed.
\end{abstract}

\section{INTRODUCTION}

Designers of lightning protection system (LPS) need to evaluate the lightning incidence of the structure to be protected. For instance, such an information is of crucial importance for engineers who must select the insulation level of an overhead power line against lightning flashover rate, in order to meet the reliability criteria set for the system [1-4].

For such an evaluation, only first strokes of negative downward flashes are generally taken into account in view of the following reasons: i) upward flashes occur mainly from very tall structures or mountain-top installations; ii) the majority of downward flashes are of negative polarity (except for tall structures and in the few regions with frequent winter thunderstorms) and iii) the majority of subsequent return stroke peak currents are smaller than the corresponding first stroke one [5].

The lightning incidence of a vertical structure is estimated by means of its so-called lightning exposure, which is related to the physical processes involved in the final stage of the progression of the downward lightning leader to the structure and of upward streamer injection. In general, in this type of studies, the downward motion of a lightning leader approaching ground is assumed to continue unperturbed, unless critical field conditions develop, allowing a juncture with a nearby vertical object generally called final jump.

Several researchers have contributed to the development of engineering models aimed at representing this complex phenomenon; nowadays it is generally accepted that the models applied to calculate lightning incidence on transmission can be grouped in two main categories:

- conventional models based on the so-called electrogeometric model (EGM) (e.g. [6-8]), which are based on the preliminary work of Golde [9];

- more recent models based on the simulation of the leader progression (LPM, [10-12]) and other similar approaches (e.g. [13-15]).

In addition to the above mentioned approaches, it is worth mentioning that a more recent representation of the lightning upward connecting leader inception has been proposed in [16] and a specific discussion about the final connecting stage between downward and upward leaders has been presented [17].

As known, the basic concept of the EMG is that it takes into account a downward lightning leader only, without taking into consideration the upward (positive) leader from the structure. Additionally, it assumes that the downward leader channel is perpendicular to the ground plane and that the flash will stroke the tower if its prospective ground termination point lies within the socalled 'attractive radius'. The attractive radius depends on several factors, such as: charge of the leader, its distance from the structure, type of structure (vertical mast or horizontal conductor), structure height.

On the other hands, the LPM has been developed from knowledge of discharge physics on long air gaps under switching surge conditions with the hypothesis of a good similarity between propagation and inception of downward and upward leaders at laboratory tests and lightning phenomena in spite of the 10x difference in scale. In [10-12] the downward propagation of the leader is determined using the known charge simulation method [18] in which fictitious line charges, which allow to provide particular solutions of Laplace and Poisson's equations satisfying specific boundary conditions, are used to calculate the leader electric field at any point. 
The use of the charge simulation method for the solution of the LPM can be replaced by numerical methods like Finite Difference (FD) or Finite Elements (FEM), which allow a more straightforward treatment of:

- boundary conditions of the problem;

- structure exposition;

- leader charge positions;

- ascending leader starting point;

- presence of non-flat terrain.

On the base of these advantages, the paper presents a numerical algorithm based on the use of the FEM [19] for the LPM calculation. In particular, the paper aims at providing the details relevant to the problem formulation and boundary condition treatment. In this respect, it is worth noting that all the general assumptions used in the original LPM were maintained in the LPM-FEM simulations.

Comparison with results obtained by solving the LPM by means of the charge simulation method (e.g. Dellera and Garbagnati [10-12]) are also provided and discussed.

The structure of the paper is the following: section 2 illustrates the problem formulation with reference to the representation of the various domains, boundary conditions, earthed structure, downward and upward leader as well as the final jump. Section 3 illustrates the results obtained by applying the proposed LPM-FEM model to a $30 \mathrm{~m}$ vertical structure as well as their comparison with the results obtained in [10-12]. Section 4 concludes the paper with the main final remarks.

\section{PROBLEM FORMULATION}

\subsection{General aspects related to the main equations and boundary conditions}

As discussed in [10-12], the representation of both upward and downward leaders propagation is determined by means of the solution of subsequent steady-state electrostatic problems. In the considered domain, the leaders charges represent the source of the problem and the earthed structure, together with the ground zeropotential and the cloud charge distribution, the boundary conditions.

As known, the problem consists in the calculation of the electrostatic field into a dielectric material region (air) $\left(\Omega \in \mathfrak{R}^{3}\right)$ provided by the solution of the Poisson's equation:

$$
-\nabla\left(\varepsilon_{0} \nabla \mathbf{V}-\mathbf{P}\right)=\rho
$$

where, $\mathbf{V}$ is the electric scalar potential, $\varepsilon_{0}$ is the permittivity of vacuum (indeed, we assume that the material in which the problem is formulated is air), $\mathbf{P}$ is the electric polarization and $\rho$ the space charge density.

The adopted FEM model solves (1) as a function of the so-called dependent variables (namely, the variables that the partial differential equation (1) is formulated for) that, in our problem formulation, are the three components $V_{x}$,
$V_{y}$ and $V_{z}$ of the electric scalar potential $\mathbf{V}$ [20].

In correspondence of the exterior boundaries $\partial \Omega$ that define $\Omega$, the following different conditions are of interest for the LPM solution:

- electric potential: $\mathbf{V}_{\partial \Omega}$;

- ground, $\mathbf{V}_{\partial \Omega}=0$;

- surface charge density: $\rho_{\partial \Omega}$;

- zero charge: $\rho_{\partial \Omega}=0$.

Concerning the interior boundaries of $\Omega$, as we assume such a domain to be characterized by a uniform dielectric medium (air), two possible conditions are of interest, namely: i) representation of leaders charges $\rho$ by means of linear distributed charge densities $\rho_{\partial^{2} \Omega}$ and ii) representation of the earthed structure by means of grounded edges $V_{\partial^{2} \Omega}=0$.

\subsection{Main domain representation}

The domain $\Omega$ (see Fig. 1) is divided into two subdomain: the first one, $\Omega_{1}$, consists of a cylindrical region of air characterized by $5 \mathrm{~km}$ radius and $2 \mathrm{~km}$ height; the second one, $\Omega_{2}$, is still a cylindrical region of air but of a reduced size in order to surround the earthed structure. As both upward leader and final jump takes place into a region of space close to the earthed structure, the aim of $\Omega_{2}$ is to provide a control volume in which a more refined finite-element mesh size can be applied. $\Omega_{2}$ is characterized by the following dimensions: $0.5 \mathrm{~km}$ radius and $0.75 \mathrm{~km}$ height.

In [10-12], the cloud charge $\left(Q_{c}\right)$ has been assumed composed by different concentric charge rings, located at $2 \mathrm{~km}$ height, aimed at providing a simplified representation of a constant surface charge density. In our simulation such a source has been directly represented by means of a constant surface charge density in correspondence of the top circular boundary of $\Omega_{1}$ (see Fig. 1).

As known, the values of $Q_{c}$ present a large dispersion (e.g. $[21,22])$. In our simulations it has been assumed $Q_{c}=-4 \mathrm{C}$ [10-12] and the relevant constant surface charge density $\rho_{\partial \Omega}$ is therefore given by the following:

$$
\begin{aligned}
& \int_{\partial \Omega} \rho_{\partial \Omega}=Q_{0} \stackrel{\rho_{\alpha 2}=\text { const. }}{\longrightarrow} \rho_{\partial \Omega}=\frac{-4 C}{\pi \cdot\left(5 \cdot 10^{3} \mathrm{~m}\right)^{2}}= \\
& =-5.093 \cdot 10^{-2}\left[\mu \mathrm{C} / \mathrm{m}^{2}\right]
\end{aligned}
$$

In Fig. 1 the top surface of $\Omega_{1}$ is a boundary condition in which a constant charge density, with value $\rho_{\partial \Omega}$ provided by (2), has been assumed.

Concerning the side surfaces of $\Omega_{1}$, we have assumed a boundary condition that allows to unconstraint the electric scalar potential, such a condition is satisfied assuming $\rho_{\partial \Omega}=0$ (see Fig. 1). 


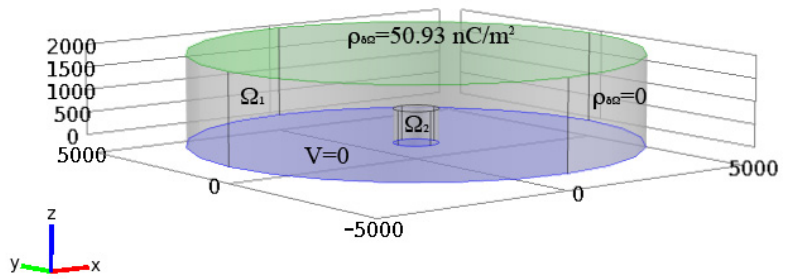

Fig. 1 Representation of domains $\Omega_{1}$ and $\Omega_{2}$ and external boundaries.

\subsection{Earthed structure representation}

In order to compare the results with those published in [10-12], we have assumed the earthed structure represented by a thin wire vertical mast. Such a structure is an interior boundary of $\Omega_{2}$ in which we have imposed a grounded $(\mathbf{V}=0)$ edge. It is worth observing that, compared to the charge simulation method, the direct adoption of such a boundary condition avoids the use of the so-called virtual charges, used in the original LPM, in order to obtain a profile of the zero-level electric potential as close as possible to the earthed structure. It is also important to note that, in principle, the charge simulation method would require a continuous modification of the virtual charges (both position and value) as the constant electric potential profiles in correspondence of the earthed structure evolves with the progression of downward and upward leaders. On the other hands the adoption of a boundary condition that forces the potential of the earthed structure to zero inherently allows to obtain a more correct representation of the problem.



Fig. 2 Voltage profiles and electric field streamlines into the $y-z$ plane of Fig. 1 for a grounded $30 \mathrm{~m}$ high vertical earthed structure, assuming as source the cloud charge only.

Fig. 2 shows the voltage profiles and the electric field streamlines into the y-z plane of Fig. 1 for a $30 \mathrm{~m}$ height vertical earthed structure assuming, as a source, the cloud $Q_{c}$ charge only.

\subsection{Downward leader representation and propagation criteria}

The downward leader is represented by means of a linear charge distribution which value is determined as the ration between the total charge correlated to the lightning current, $Q_{f p}$, and the average total leader length of $2 \mathrm{~km}$ (distance between the simplified cloud charges and the ground, that corresponds to the height of $\Omega_{1}$ ). In order to compare the results with those of [10-12], we have adopted the same expression that provides the average value of $Q_{f p}$ as a function of the lightning waveshape first peak current expressed in kA [23,24]:

$$
Q_{f p}=76 \cdot I^{0.68} \cdot 10^{-3}[C]
$$

Therefore, in correspondence of the interior boundary $\rho_{\hat{\partial}^{2} \Omega}$ of $\Omega_{1}$ that simulates the downward leader, the following condition has been imposed:

$$
\rho_{\partial^{2} \Omega}^{\text {down }}=38 \cdot I^{0.68}[\mu \mathrm{C} / \mathrm{m}]
$$

It is worth adding that, in agreement with [10-12], the most advanced part of the downward leader (leader tip) is characterized by a value of linear charge density $\rho_{\partial^{2} \Omega}^{\text {down }}$ uncorrelated to the lightning current and assumed equal to $100 \mu \mathrm{C} / \mathrm{m}$.

As known, the propagation direction of the downward leader depends to the phenomenon associated to the negative streamers that start from the leader tip (e.g. [16]). These streamers tends to follow the streamlines of the electric fields and, therefore, like in the original LPM, it has been assumed that the direction of the downward leader corresponds to the one of the maximum gradient of the electric potential, namely the direction of the maximum electric field streamline, estimated in correspondence of a domain in front of the streamer zone. The extension of such a domain is assumed equal to a region in front of the downward leader tip in which the electric field is above $300 \mathrm{kV} / \mathrm{m}$.

In view of the above, the estimation of the maximum gradient of the electric potential is performed in correspondence of a hemisphere, centered in correspondence of the leader tip, which radius can range from few tens to few meters (more precisely from $20 \mathrm{~m}$ to $3 \mathrm{~m}$ ) as a function of the streamer zone extension. Fig. 3a shows the top view of the electric potential in correspondence of such a hemisphere ( $20 \mathrm{~m}$ diameter) for a downward leader corresponding to a peak current of 20 $\mathrm{kA}$ at $50 \mathrm{~m}$ from the ground and $25 \mathrm{~m}$ from the vertical structure. Fig. $9 \mathrm{~b}$ shows the same distribution but making reference to the spherical coordinate system that parameterize the hemisphere as a function of the latitude $(\vartheta)$ and longitude $(\varphi)$. The white zone in Fig. $9 \mathrm{~b}$ indicates the region corresponding to the maximum electric field gradient. It can be noted in Fig. 3a the asymmetrical distribution of the electric potential as well as, in Fig. 3b, 
the relevant maximum gradient region located into $(\vartheta, \varphi)$ coordinate different from $(0, \pi)$.



a)

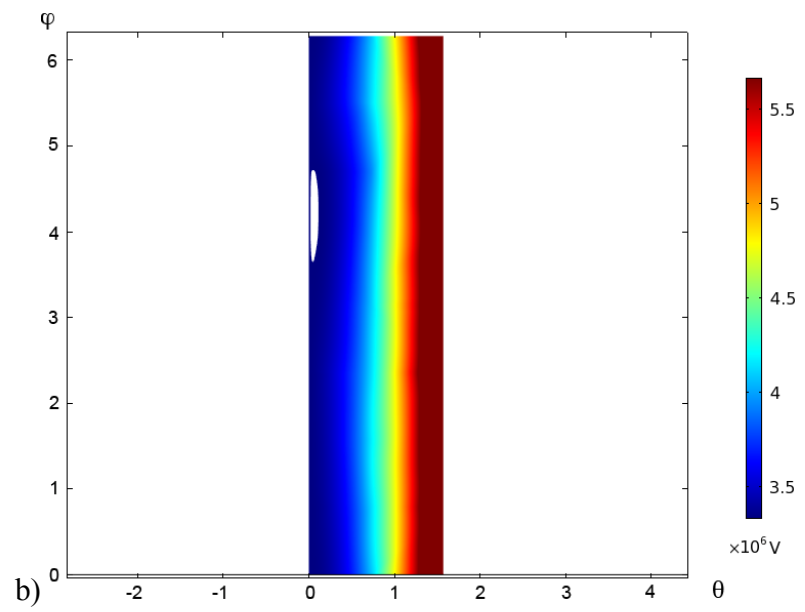

Fig. 3 Electric potential profiles in correspondence of the hemisphere control surface with $20 \mathrm{~m}$ diameter for a downward leader corresponding to a peak current of $20 \mathrm{kA}$. Leader tip at $50 \mathrm{~m}$ from the ground and $25 \mathrm{~m}$ from the earthed structure. a) top view in the $x y$ plane of the hemisphere control surface electric potential; b) electric potential of the hemisphere represented in spherical coordinate system.

Fig. 4 shows the electric potential iso-surfaces associated to a downward leader corresponding to a peak current of $20 \mathrm{kA}$. At this stage of the simulation (downward leader at $360 \mathrm{~m}$ from ground) the inception conditions for the formation of the upward leader from the $30 \mathrm{~m}$ structure have not yet been reached (see next paragraph). It can be seen the deformation of the electric potential iso-surfaces due to: i) the different linear charge density of the leader channel compared to the leader tip $\left(1.5 \cdot 10^{7} \mathrm{~V}\right.$ iso-surface) and ii) the presence of the earthed structure $\left(0.01 \cdot 10^{7} \mathrm{~V}\right.$ iso-surface).

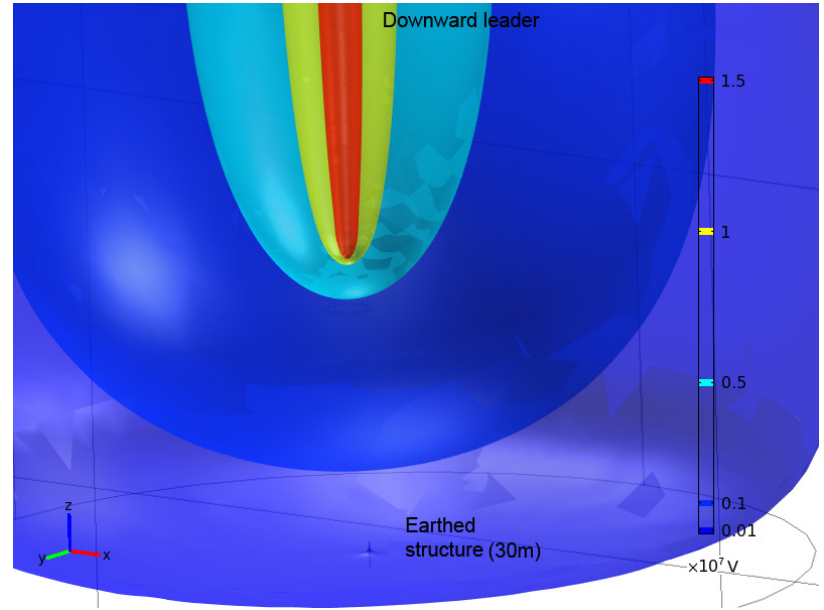

Fig. 4 Electric potential iso-surfaces associated to a downward leader corresponding to a peak current of $20 \mathrm{kA}$. Downward leader at $360 \mathrm{~m}$ from the ground.

\subsection{Upward leader representation and propagation criteria}

This section illustrates and discusses two main aspects concerning the upward leader, namely: inception and consequent propagation.

As discussed in the introduction, the LPM is based on the similarity between laboratory tests and lightning phenomena of the propagation and inception of downward and upward leaders. Concerning the upward leader inception, on the basis of the observations of $[25,26]$, Dellera and Garbagnati assume that the conditions for the inception of positive leaders from earthed structures is substantially not influenced by the earthed electrode size, up to the so called 'critical radius'. The critical radius, assumed in [10-12] in the order of few tens of centimeters, defines therefore a region of space surrounding the earthed structure above which we can calculate the electric field in order to determine the upward leader inception. Such a calculation has been implemented by means of a so-called 'control-surface' $\Sigma$ that surrounds the earthed structure. Fig. 5 shows the norm of the electric field in correspondence of $\Sigma$ with the same conditions used to obtain Fig. 4.

For each point $P(x, y, z) \in \Sigma$ the value of the norm of the electric field is compared to the threshold value that provides the upward leader inception. Compared to the model of Dellera and Garbagnati, in which the starting point of the upward leader was assumed a-priori in correspondence of the position of the virtual charges of the charge-simulation method, the model here presented allows the estimation of the inception position into the whole area that surrounds the earthed structure.

Concerning the inception critical field, in order to provide a comparison with the same conditions of [1012], we have adopted the value derived from [2] and reported for convenience in Fig. 6. 


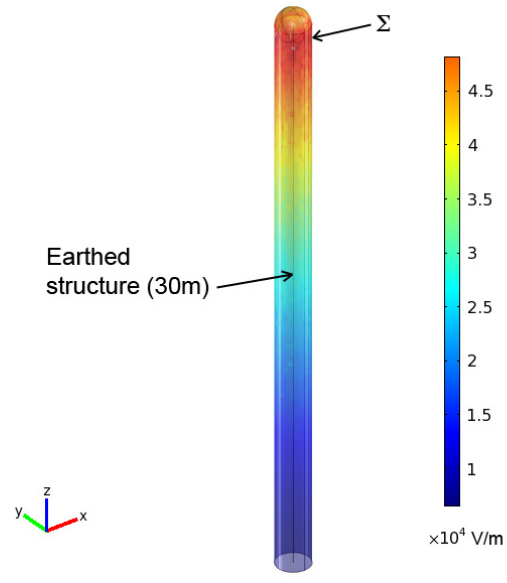

Fig. 5 Norm of the electric field in correspondence of the control surface surrounding the earthed structure. Downward leader corresponding to a peak current of $20 \mathrm{kA}$ located at 360 $\mathrm{m}$ from the ground.



Fig. 6 Critical leader inception electric field as a function of the electrode radius. Adapted from [2].

Concerning the upward leader propagation, it has been assumed that the ratio between downward and upward leader propagation speeds is constant and equal to 4 . Therefore, as the spatial propagation steps of the downward leader are of $10 \mathrm{~m}$, the corresponding upward leader ones are of $2.5 \mathrm{~m}$. The upward positive leader charge has been assumed independent from the downward leader charge and equal to $50 \mu \mathrm{C} / \mathrm{m}$. The criteria used for the calculation of the propagation direction of the upward leader are the same of the ones adopted for the downward leader.

\subsection{Final jump}

The progressive propagation of downward and upward leaders could evolve into a so-called final if, in the space region between the two leaders, it is possible to identify a path characterized by a voltage gradient larger than the streamer gradient value, namely an electric field in the order of $500 \mathrm{kV} / \mathrm{m}$ (e.g. $[13,17])$

Such a path estimation has been implemented as follows: for each electric field streamline connecting the two leaders it has determined whether the electric field exceeds the value of $500 \mathrm{kV} / \mathrm{m}$ along the overall streamline length. Fig. 7 shows an example of such an estimation concerning the case of a downward leader corresponding to a peak current of $20 \mathrm{kA}$ located at $23 \mathrm{~m}$ from the earthed structure. In particular, Fig. 7a shows the electric field norm iso-surfaces together with the electric field streamlines, Fig. $7 \mathrm{~b}$ shows the propagation of the upward and downward leaders together with the final jump path. In Fig. 7a is evident a region where the two iso-surfaces of electric field norm equal to $500 \mathrm{kV} / \mathrm{m}$ are touching each other. In such a region, the final jump can take place as shown by the result of Fig. $7 \mathrm{~b}$.

a)

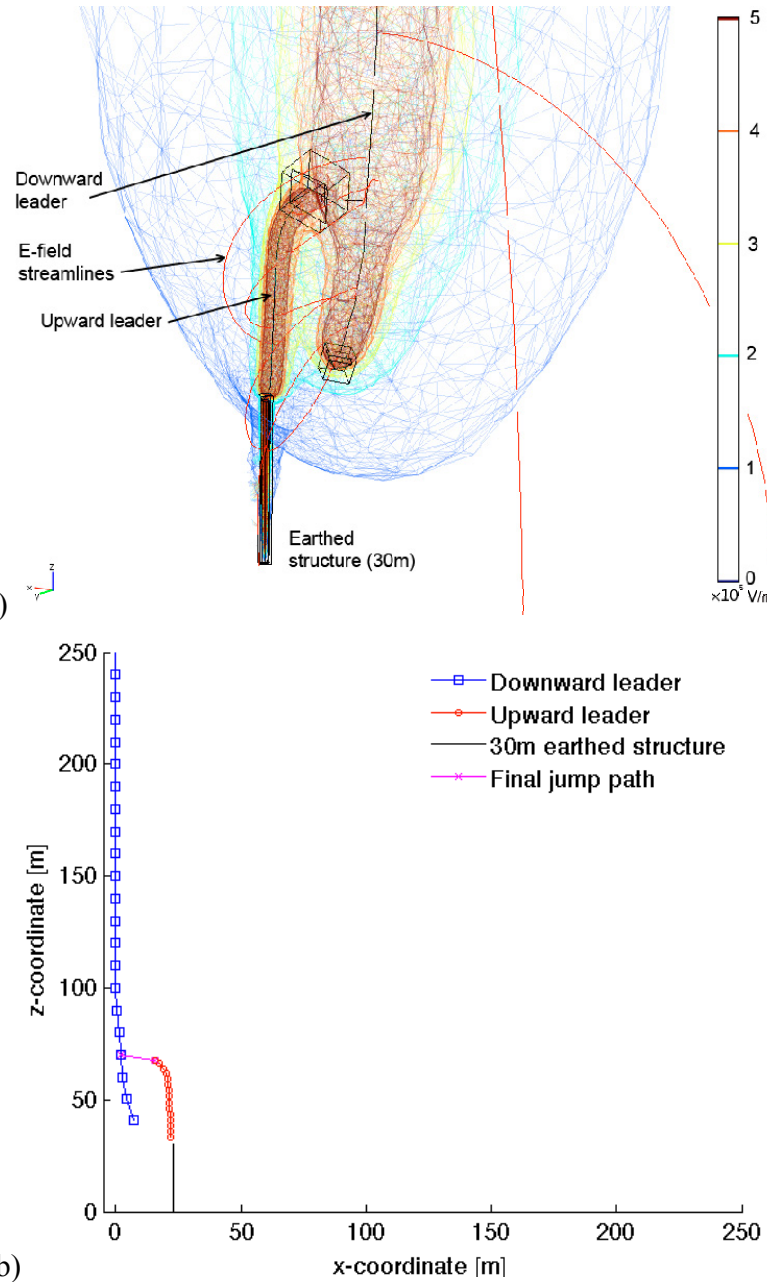

Fig. 7 Final jump estimation concerning the case of a downward leader corresponding to a peak current of $20 \mathrm{kA}$ located at $23 \mathrm{~m}$

from the $30 \mathrm{~m}$ high earthed structure: a) electric field isosurfaces and streamlines, b) downward/upward leaders and final jump paths.

\section{RESULTS AND COMPARISONS}

The procedure to determine the lateral distance for a specific earthed structure and lightning current is the following: 
1. definition of the starting point of the downward leader $P_{d l, 0}\left(x_{0}, y_{0}, z_{0}\right)=(\tilde{x}, 0,2000) \quad$ where subscript ' 0 ' indicates the initial step of the leader propagation and $\tilde{x}$ the distance between the downward leader projection on the $x y$ plane and the earthed structure supposed located in the center of $\Omega$ (in the first simulation $\tilde{x}=0$, namely the downward leader descends in correspondence of the earthed structure);

2. leaders progression simulation in order to determine whether one of the two following conditions is verified: i) the two leaders are touching each other, ii) a final jump takes place;

3. shift of the starting point of the downward leader by increasing the $\tilde{x}$ coordinate, and iterative repetition of points 1 and 2 .

When condition 2 is not verified for the first time during the process, the corresponding $\tilde{x}$ defines the lateral distance.

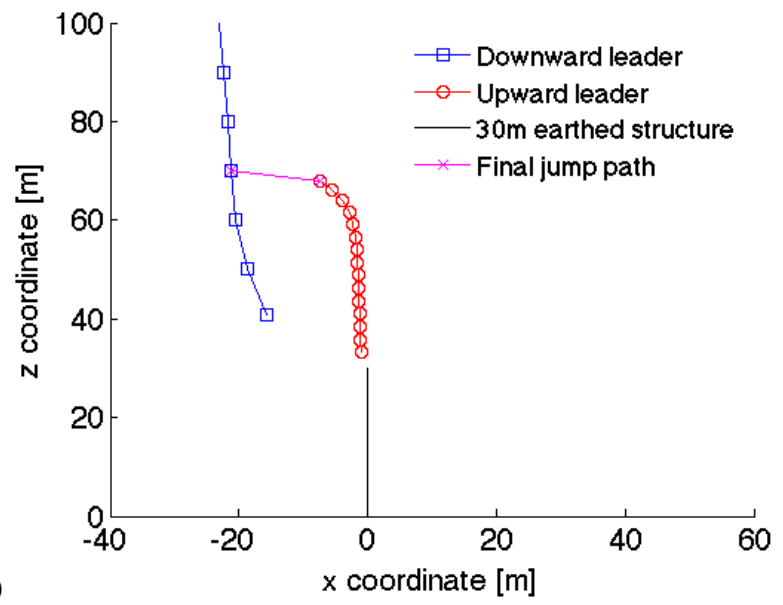

a)

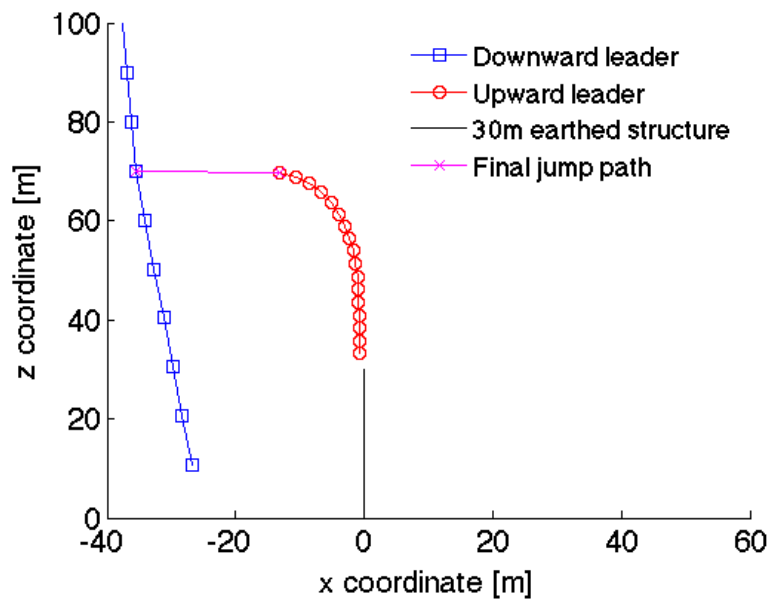

Fig. 8 shows an example of the lateral distance assessment concerning the case of a $20 \mathrm{kA}$ current for $\tilde{x}=23 \mathrm{~m}, 36 \mathrm{~m}, 37,5 \mathrm{~m}$ and $39.5 \mathrm{~m}$.

In order to compare the results obtained with the proposed LPM-FEM model with those of [10-12], we have made reference to the case of a $30 \mathrm{~m}$ structure for which we have determined the lateral distances in correspondence of the lightning currents of 10,20,50 and 100 kA. Fig. 9 shows such a comparison. As it can be seen, in the low current region the LPM-FEM essentially provides the same results of the original version of the LPM, whilst for currents larger than $50 \mathrm{kA}$ the results of the LPM-FEM tends to result into lower lateral distances. The results obtained with the LPM-FEM seems to be in agreement with other lateral distance expressions provided in the literature that predicts a non-linear dependency of the lateral distance with the lightning current.

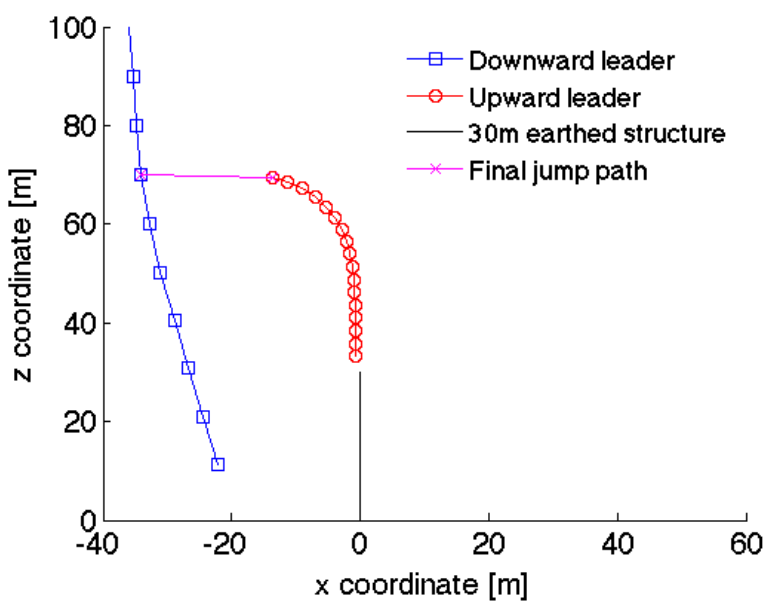

b)

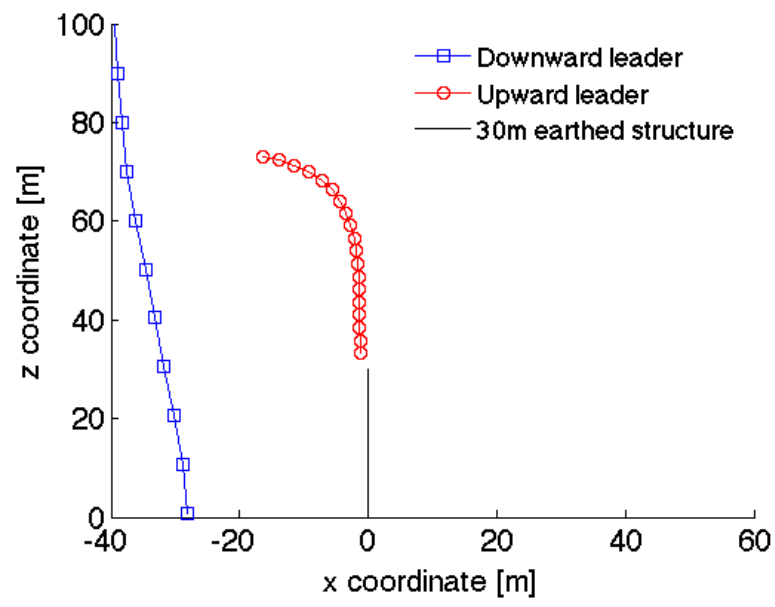

Fig. 8 Lateral distance estimation for the case of a $30 \mathrm{~m}$ high earthed structure and $20 \mathrm{kA}$ lightning current: a) $\tilde{x}=23 \mathrm{~m}, \mathrm{~b}) \tilde{x}=36 \mathrm{~m}$, c) $\tilde{x}=37.5 \mathrm{~m}, \mathrm{~d}) \tilde{x}=39.5 \mathrm{~m}$. 


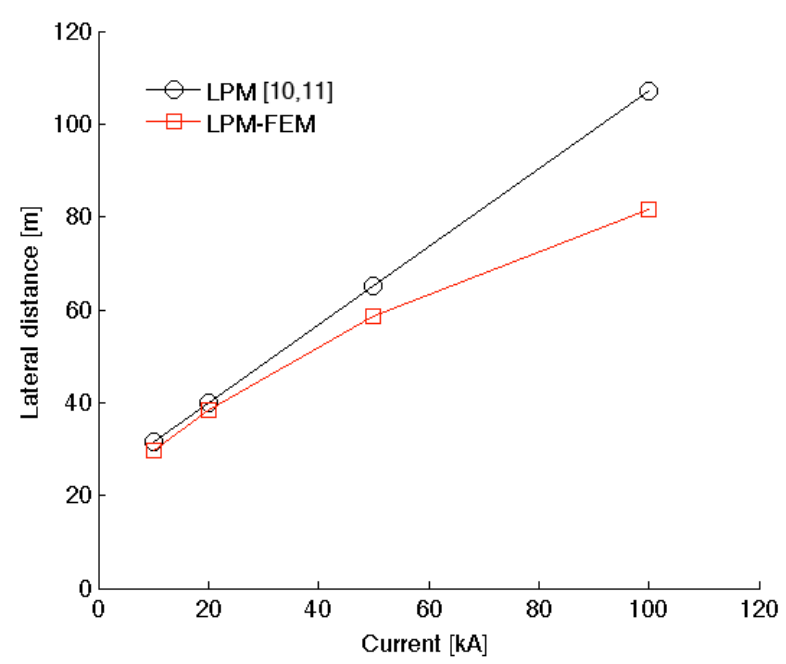

Fig. 9 Comparison between lateral distances of [10-12] with the ones obtained with the LPM-FEM, $30 \mathrm{~m}$ high earthed structure.

\section{CONCLUSIONS}

The paper has proposed the solution of the leader progression model by means of the finite element method. Compared with the charge simulation method, the one here proposed allows to straightforward take into account the boundary conditions of the problems. This avoids the use of virtual charges, needed in [10-12] to find the solution of the electrostatic field distribution, resulting into the following advantages: i) the electric scalar potential in correspondence of the earthed structure is inherently null as it is a boundary condition of the problem, ii) the inception of the upward leader can be evaluated in correspondence of any point of the earthed structure and even multiple upward leaders can be taken into account.

In view of the numerical solution of the electrostatic problem provided by the FEM, it also presents the following further advantages:

- representation of any charge distribution for both upward and downward leaders;

- implementation of different final jump criteria;

- implementation of different inception models;

- representation on any 3D earthed structure (of interest for asymmetrical structure configurations).

Future works will be devoted to the possible numerical evaluation of the streamer zone extension (in front of the leader tips) by means of a representation the non-linear properties of the medium.

\section{ACKNOLEGMENTS}

The authors wish to thank CESI s.p.a., for providing the access to the original LPM code and the share of the LPM contents.

Thanks are also due to D. Sbrega for his help in performing the calculations.

\section{REFERENCES}

[1] CIGRÉ WG 33-01, "Guide to Procedures for Estimating the Lightning Performance of Transmission Lines", Cigré Brochure 63, October 1991.

[2] CIGRÉ Task Force 33-01-03, "Lightning Exposure of Structures and Interception Efficiency of Air Terminale", Cigré Brochure 118, October 1997.

[3] IEEE Std. 1243, "IEEE Guide for Improving the Lightning Performance of Transmission Lines", June 26, 1997.

[4] IEEE Std 1410-2004 "Guide for improving the lightning performance of electric power overhead distribution lines", 2004.

[5] R. B. Anderson and A. J. Eriksson, "Lightning parameters for engineering applications," Electra, no. 69, pp. 65-102, Mar. 1980.

[6] F.S. Young, J. M. Clayton and A. R. Hileman, "Shielding of Transmission Lines," IEEE Trans. on PAS, vol. 82, pp. 132-154, 1963.

[7] G.W. Brown and E. R. Whitehead, "Field and Analytical Studies of Transmission Line Shielding", IEEE Transactions on PAS, vol. 88, pp. 617-626, 1969.

[8] A.M. Mousa and K.D. Srivastava, "The implications of the electrogeometric model regarding effect of height of structure on the median amplitude of collected lightning strokes", IEEE Trans. PowerDelivery, vol. 4, no. 2, pp. $1450-1460,1989$.

[9] R.H. Golde, "The Frequency of occurrence and the distribution of lightning flashes to transmission lines", AIEE Trans., 64, pp. 902-910, 1945.

[10] L. Dellera, E. Garbagnati, "Lightning stroke simulation by means of the leader progression model (Part I)". IEEE Trans. on PWRD, Vol.5, No.4. November 1990.

[11] L. Dellera, E. Garbagnati,"'Lightning stroke simulation by means of the leader progression model (Part II)". IEEE Trans. on PWRD, Vol. 5, No. 4. November 1990.

[12] M. Bernardi, L. Dellera, E. Garbagnati , G. Sartorio, “ Leader Progression Model of Lightning: Updating of the Model on the basis of recent Tests Results", Proc. of the $23^{\text {rd }}$ International Conference on Lightning Protection (ICLP), September 1996.

[13] F.A.M. Rizk, "Modeling of Transmission Line Exposure to Direct Lightning Strokes", IEEE Trans. on Power Delivery, Vol. PWRD-5, No.5, October 1990, pp 19831997.

[14] F.A.M. Rizk, "Modelling of Lightning Incidence to tall structures. Part I: Theory". IEEE Trans. on PWRD, Vol. 9, No. 1, January 1994.

[15] M. Vargas, V. Cooray, M. Becerra, H. Torres, "Proc. of the 23rd International Conference on Lightning Protection (ICLP), September 1996.”, Proc. of the $28^{\text {th }}$ International Conference on Lightning Protection (ICLP), September 2006.

[16] M. Becerra and V. Cooray, "A Simplified Physical Model to Determine the Lightning Upward Connecting Leader Inception”, IEEE Trans. on PWRD, vol. 21-2, 2006, pp. 897-908.

[17] F. A. M. Rizk, "Modeling of Substation Shielding Against Direct Lightning Strikes", in press on IEEE Trans. on EMC, 2010.

[18] H. Singer, H. Steinbigler, P. Weiss, "A Charge simulation method for the calculation of High Voltage fields", IEEE 
PES Winter Meeting, January 1974.

[19] J. Jin, "The Finite Element Method in Electromagnetics", Wiley-IEEE Press, $2^{\text {nd }}$ edition, May, 2002.

[20] COMSOL MULTIPHYSICS v. 3.3, AC/DC module library, august 2006.

[21] M. Brook, T. Ogawa, "The cloud discharge", Chapter 6 of Volume 1 "Physics of lightning", Book edited by R.H. Golde, Academic Press, London 1977.

[22] V. Rakov and M.A Uman, "Lightning Physics and Effects", Cambridge University Press, UK, 2003.

[23] K. Berger "The earth flash", Chapter 5 of Volume 1 "Physics of lightning" Book edited by R.H. Golde,
Academic Press, London 1977.

[24] K. Berger: E. Vogelsanger, "Measurements and Results of Lightning Records at Monte S. Salvatore from 1955 to 1963", In German, Bull. SEV, 1965, vol. 56, N. 1, pp. 222.

[25] Les Renardières Group, "Long Air Gap Discharges at Les Renardières: 1973 Results", Electra, No. 23, July 1972 and No. 35, July 1974.

[26] G. Carrara, L. Thione, "Switching surge strength of large air gaps: a physical approach", IEEE Trans., Vol. PAS-95, No.2, pp. 512-524, March/April, 1976. 